\title{
Sur diverses citations, et notamment sur trois passages de Malalas retrouvés dans un texte hagiographique.
}

La troisième des Passions de sainte Catherine d'Alexandrie publiées en 1897 par M. Viteau, renferme des discours pleins de citations. ${ }^{1}$ ) Diodore, Plutarque, Homère, Orphée, Sophocle, Platon, un oracle d'Apollon, sont invoqués soit par le rhéteur qui essaie de confondre la sainte, soit par la sainte qui réussit à confondre le rhéteur. Personne n'a encore, que je sache, déterminé l'origine de toute cette érudition. Elle remonte, pour une très grande partie, ou bien à Malalas, ou bien à l'un des documents dont le texte de Malalas est dérivé. Avant d'aborder le premier des passages où se révèle cette filiation, il faut noter que la troisième Passion (ou texte $\mathrm{C}$ de $\mathrm{M}$. Viteau) a été la source du remaniement attribué à Métaphraste ${ }^{2}$ ), et qu'elle nous est conservée entre autres dans un Parisinus du $\mathrm{X}^{\bullet}$ siècle. $^{3}$ )

Premier passage:

Viteau p. 45, 1. 304): $\tau \tilde{\omega} \ldots \Delta \iota{ }^{4}$ - Malalas 54, 12 (ed. Bonn.): $\pi \varepsilon \rho i$

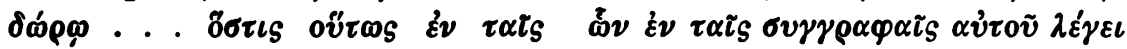

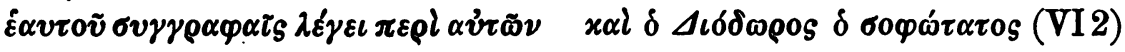

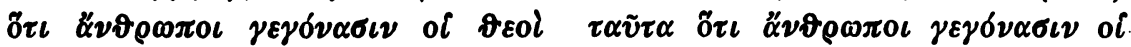

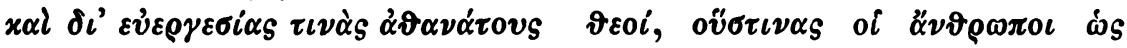

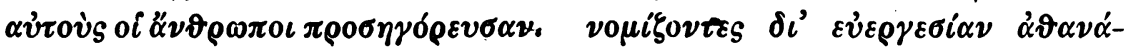

1) Passions des saints Écaterine et Pierre d'Alexandrie . ., publiées par M. J. Viteau; Paris, Bouillon, 1897; troisième terte (C), p. 44 et suiv. - Sur cette édition, roir K. K(rumbacher), Byz. $Z$. VII 482.

2) Migne, Patrol. gr., t. 116, col. 275 et suiv.

3) Parisinus 1180: cf. Catalog. cod. hagiogr. gr. Biblioth. nat. Parisiensis p. 80. Dans certains manuscrits, par exemple dans le Parisinus 2408 (XIII' s., f. 224) et dans le Marcianus 148 (XIV• 8.; cf. Viteau, ibid., p. 41), on trouve des extraits des discours adressés par la sainte au rhéteur. - Sur la filiation de ces passions, M. Viteau a publié une étude dans les Annales de S. Louis des Français III (1898), 5-23; je ne la connais que par une notice des Analecta Bollandiana XVIII, p. 69, peu rassurante sur la valeur de ses conclusions.

4) Je reproduis tel quel le texte de $M$. Viteau. 


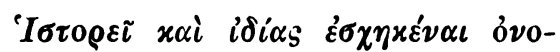

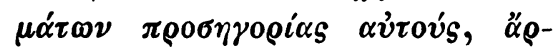

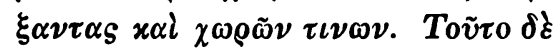

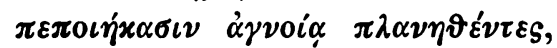

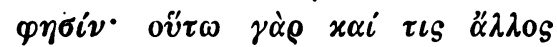

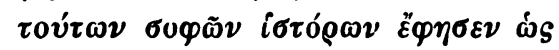

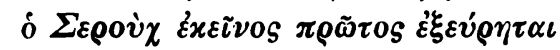

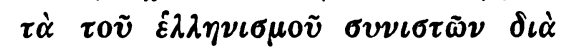

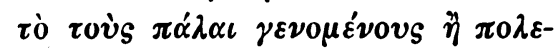

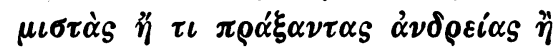

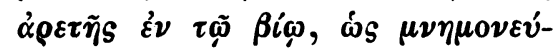
$\varepsilon \sigma \vartheta \alpha \iota{ }^{\prime 2} \xi_{\zeta} \iota \nu, \dot{\alpha} \nu \delta \rho \iota \tilde{\alpha} \sigma \iota \tau \iota \mu \tilde{\eta} \sigma \alpha \iota \sigma \tau \eta-$ $\lambda \tilde{\omega} \nu$.

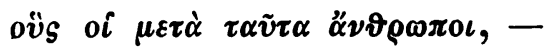

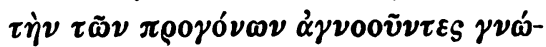

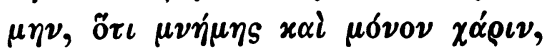

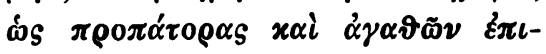

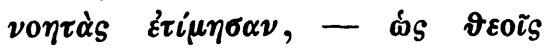

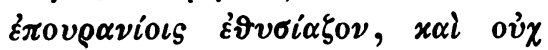

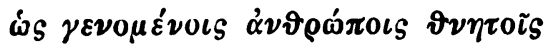

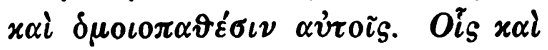

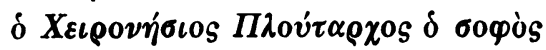
$\dot{v} \mu \tilde{\omega} \nu \quad x \alpha \tau \alpha \mu \varepsilon \dot{\mu} \mu \varphi \varepsilon \tau \alpha \iota$ ம் $\pi \lambda \dot{\alpha} \nu \eta \nu$

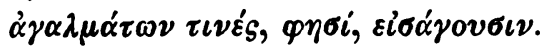

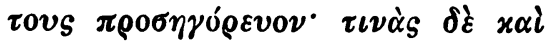

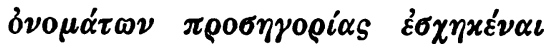

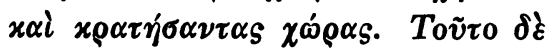

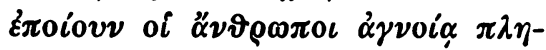

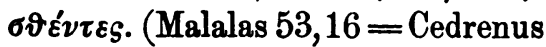

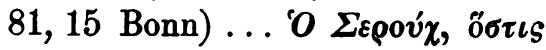

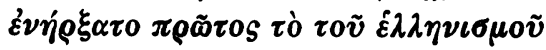

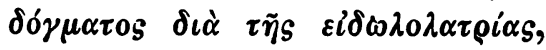

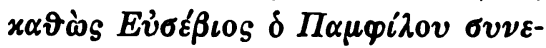

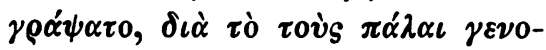

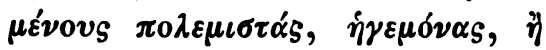

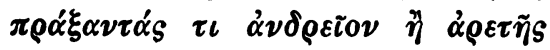

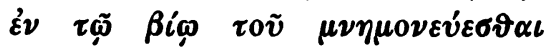

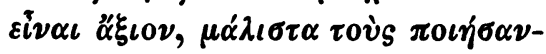

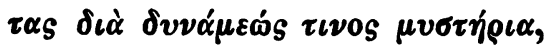

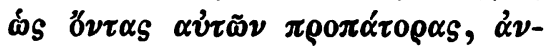
$\delta \rho i \tilde{\alpha} \sigma \iota \quad \sigma \tau \eta \lambda \tilde{\omega} \nu \varepsilon^{2} \tau i \mu \eta \sigma \alpha \nu, \ldots \ldots$

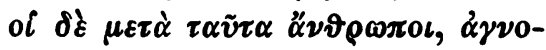

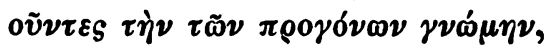

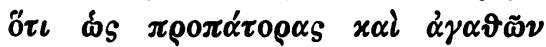

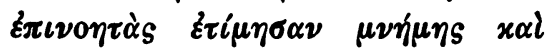

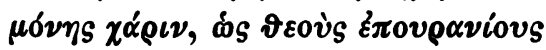

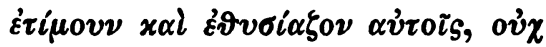

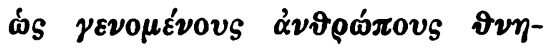

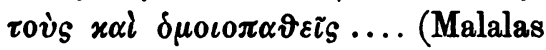
56, 3 = Cramer Anecd. Par. II

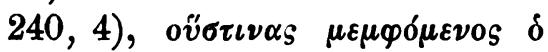

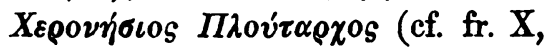
tiré d'Eusèbe, Praep. Ev., III 8)

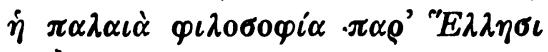

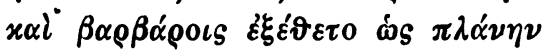

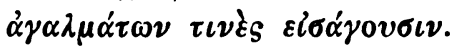

La ressemblance du discours de sainte Catherine avec la chronique de Malalas est frappante. Des deux textes, e'est parfois celui de l'hagiographe qui paraît le meilleur, même dans l'état où l'éditeur moderne nous le présente: notamment, la Passion confirme la conjecture $\pi \lambda \alpha \nu \eta \vartheta \varepsilon^{\prime} \nu \tau \varepsilon S$ (1. 4) de Wesseling. Mais laissons au futur éditeur de la Xoovoroccqía le soin de démêler ce que le texte hagiographique apporte d'intéressant. 


\section{Deuxième passage:}

Viteau p. 51, 1. 25:

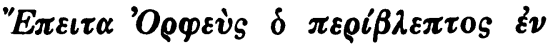

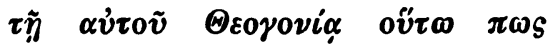
$\dot{\alpha} \pi \varepsilon v \chi \alpha \rho \iota \sigma \tau \tilde{\omega} \nu \tau \tilde{\omega}{ }^{\prime} A \pi \delta \dot{d} \lambda \omega \nu \iota$ '

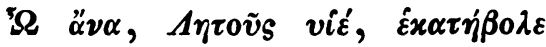

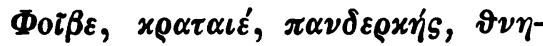

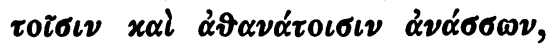

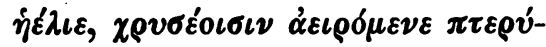


Troisième passage:

Viteau p. 51, dernière ligne: 'Ov $\delta$ है

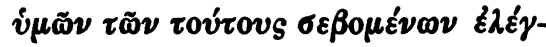

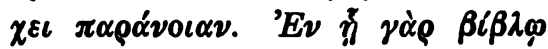

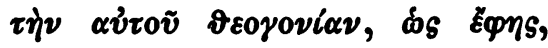

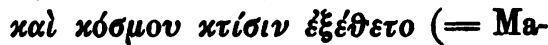

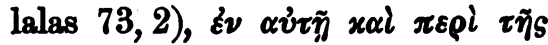

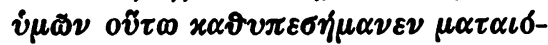

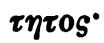

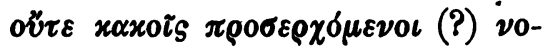
$\tilde{\eta} \sigma \alpha \iota$

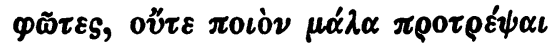

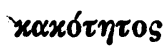

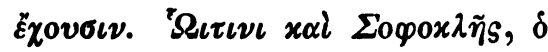

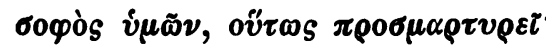

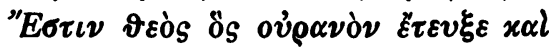

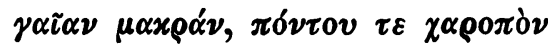

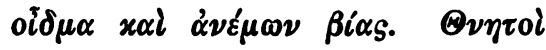

Malalas p.72,16(=Cedrenus 101,11; cf. Cramer Anecd. Par. II 241, 27):

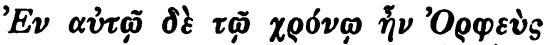

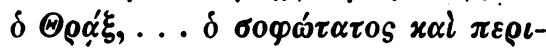

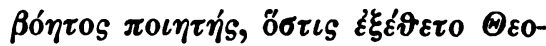

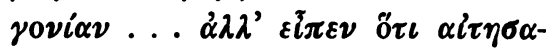

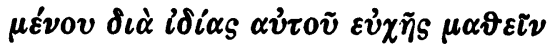

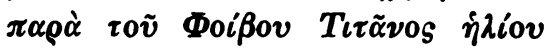

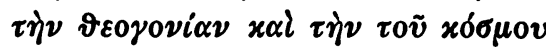

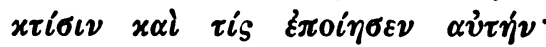

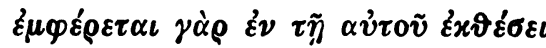

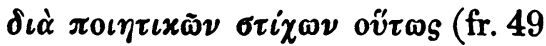
Abel).

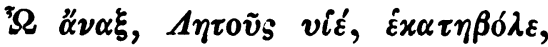

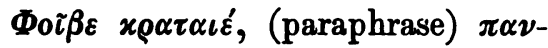

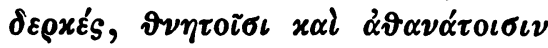

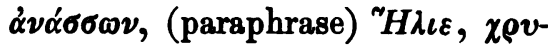

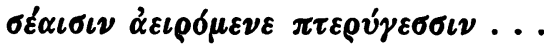

Malalas 75, $6=$ Cedrenus 103, 7 :

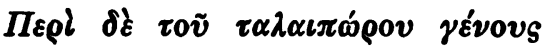

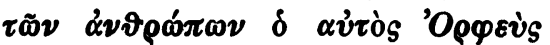

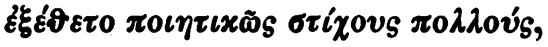

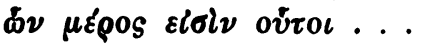

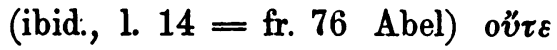

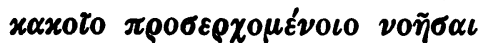

(paraphrase)

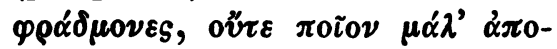

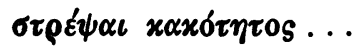

(Malalas 40, 15= Cedrenus 82, 7)

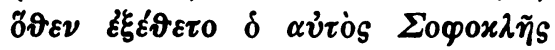
(Nauck ${ }^{8}$, fr. 1025) $\varepsilon^{2} \nu$ rois $\alpha \dot{v} \tau o \tilde{v}$

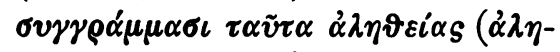

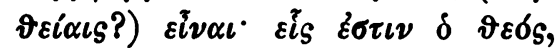

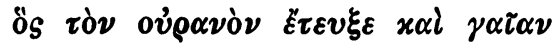




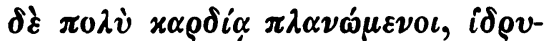
$\sigma \alpha \dot{\mu} \mu \varepsilon \vartheta \alpha \pi \varepsilon \mu \mu \alpha \dot{\tau} \tau \omega \nu \pi \alpha \rho \alpha \psi v \chi \dot{\alpha} S \Theta \varepsilon \tilde{\Phi} \nu$ $\dot{\alpha} \gamma \alpha \dot{\lambda} \lambda \mu \alpha \tau \alpha, \quad \xi \dot{v} \lambda \omega \nu \quad x \alpha i \quad \lambda i^{\prime} \vartheta \omega \nu \quad \dot{\varepsilon} x$

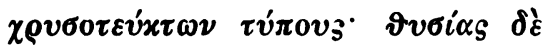

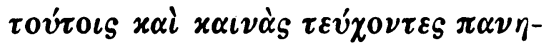


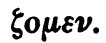

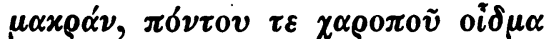

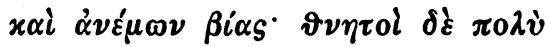

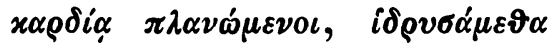
$\pi \eta \mu \alpha^{\prime} \tau \omega \nu$ ( $\pi \varepsilon \mu \mu \alpha \dot{\tau} \tau \omega \nu$ Cramer Anecd.

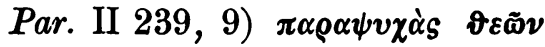

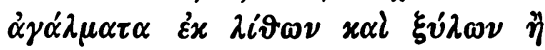

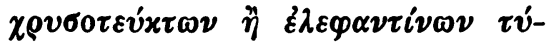

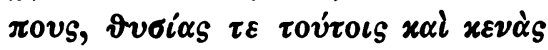

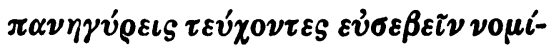
$\xi о \mu \varepsilon \nu$.

Après cette tirade, on trouve dans la Passion deux citations, l'une, d'un passage attribué à Platon, l'autre, d'un prétendu oracle d'Apollon; à la différence des précédents, ces deux textes ne se retrouvent pas dans l'édition moderne de Malalas; du moins, je les y ai cherchés en vain. Mais ils coïncident avec deux extraits édités par Bentley ${ }^{1}$ ), d'après un Baroccianus qui les donne dans un recueil inti-

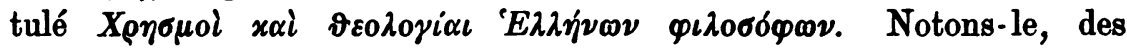
quinze morceaux qui, suivant Bentley, constitueraient ce recueil, cinq au moins se retrourent dans le texte de Malalas ${ }^{8}$ ), tel qu'il est connu aujourd'hui:

Viteau p. 53, 1. 13: Bentley p. 686:

'E

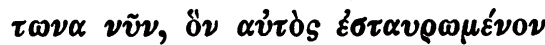

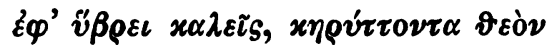

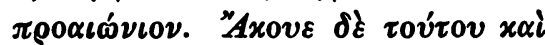

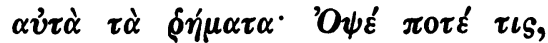
$\varphi \eta \sigma i \nu, \quad z \pi l \quad \tau \dot{\eta} \nu \pi 0 \lambda v \sigma \chi \iota \delta \tilde{\eta} \tau \alpha v^{\prime} \tau \eta \nu$

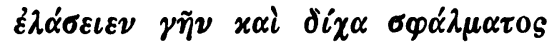

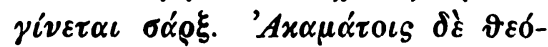
$\tau \eta \tau 0 \varsigma$ ő

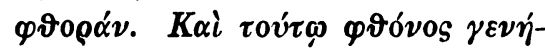

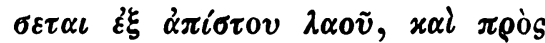

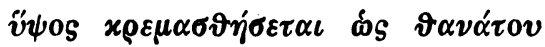

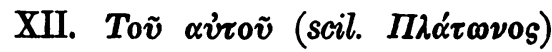
$\pi \varepsilon \rho i$ X

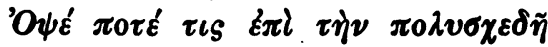

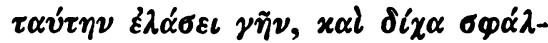

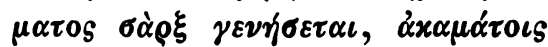

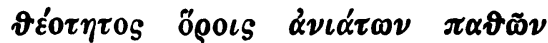

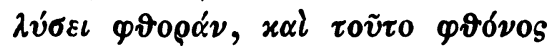

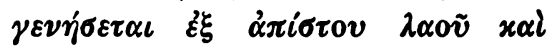

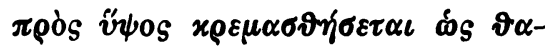

1) Richardi Bentleii epistola ad cl. v. Ioannem Millium, reproduite par l. Dindorf à la fin de son édition de Malalas; voir p. 683 de cette édition.

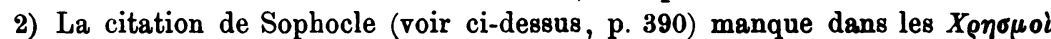
d'Oxford, mais d'après une indication de Vitelli (Nauck, trag. gr. fr. ${ }^{2}$, p. 358), ce morceau figure dans le Neapol. II F 9, f. 140v. Il est donné aussi par l'Angelicanus 43 , f. $18^{\mathrm{r}}$ (Studi italiani IV, p. 88). Je ne puis qu'appeler l'attention sur ce fait. In nous manque actuellement une analyse et un classement des

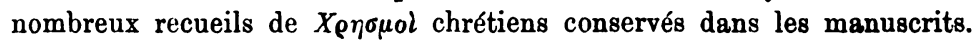




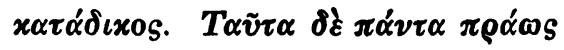

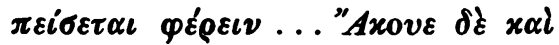

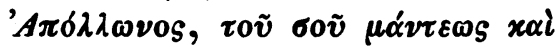

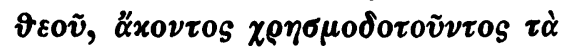

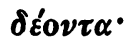

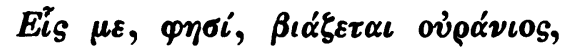

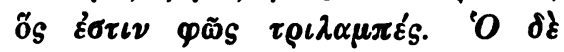

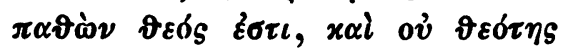

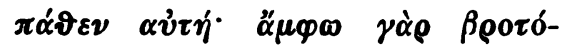

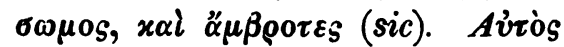

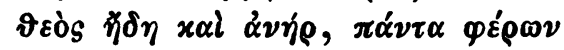

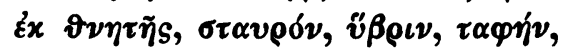

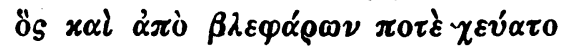

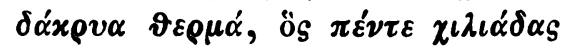

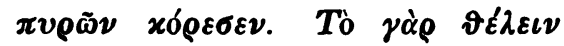

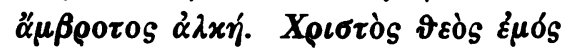

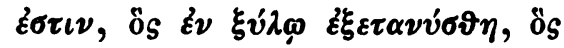

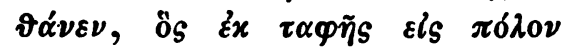
ต้อто ... .

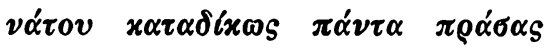
$\pi \varepsilon i \sigma \varepsilon \tau \alpha \iota$.

(Bentley, ibidem)

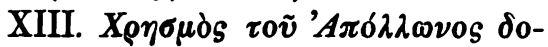

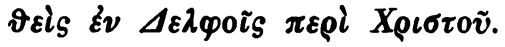

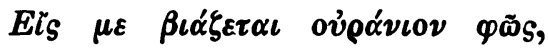

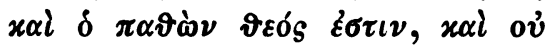

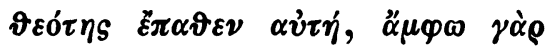

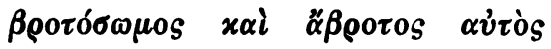

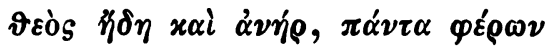

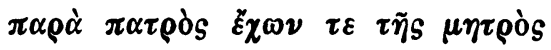

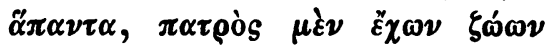

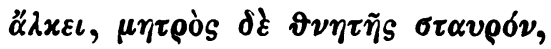

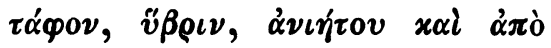
$\beta \lambda \varepsilon \varphi \alpha ́ \rho \omega \nu \pi 0 \tau \varepsilon \chi \varepsilon v \tilde{\alpha} \tau \dot{\alpha}(s i c) \delta \alpha ́ x \rho v \alpha$

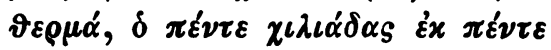

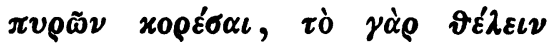

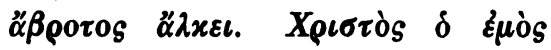

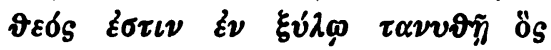

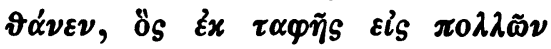

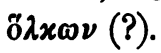

C'est là une version assez altérée, semble-t-il, d'un oracle dont on rencontre des morceaux dans beaucoup de recueils analogues à celui

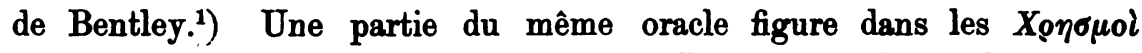

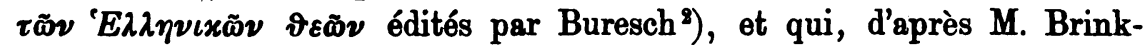
mann, serait une réduction de la @eoбopia d'Aristokritos. ${ }^{3}$ ) Le texte le plus complet et le plus correct de cet oracle a été donné par Buresch également, en appendice, d'après le manuscrit $n^{\circ} 32$ de la bibliothèque nationale d'Athènes.4) Quant à la version de l'oracle reproduite cidessus, et commune au recueil de Bentley et à la Passion de sainte Catherine, elle se retrouve encore dans une collection intitulée $\Pi_{\varepsilon \rho l}$

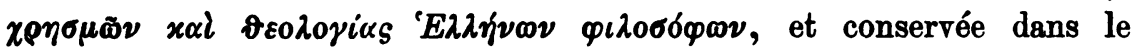
cod. Tischend. VIII (XVI ${ }^{\circ}$ siècle) de la bibliothèque de l'université de Leipzig. ${ }^{5}$ ) Je ne puis songer évidemment à déterminer ici les rapports

1) Voir notamment Wolff, Porphyrii de philosophia ex oraculis haurienda, p. 234-235 et notes.

2) Klaros, p. 95 et suiv.; Buresch semble avoir ignoré l'intéressant $\alpha \nu \xi ́ x \delta o \tau o \nu$ de Bentley.

3) Rhein. Museum, t. 51, p. 273 et suiv. 4) Klaros, p. 130-131.

5) Voir R. Volkmann, Jahrb. f. klass. Philol. t. 77 (1858), p. 870, note, et p. 876. Même version encore dans l'Angelicanus 43 (Studi ital., IV, 88). 
qui existent entre les différentes reproductions de l'oracle, ni à dire si

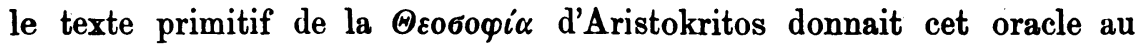
complet. Je dois me borner aussi à indiquer, sans essayer de la résoudre, la question des rapports de Malalas avec les divers recueils de X

Enfin - et ceci achèvera de mettre sous les yeux du lecteur toutes les données du problème -, il y a deux passages de la Passion de sainte Catherine qui pourraient avoir la même origine que les précédents, mais pour lesquels je n'ai pas trouvé les mêmes coïncidences:

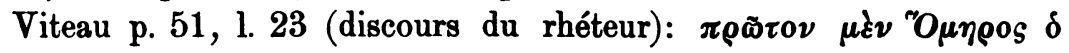

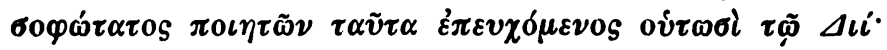

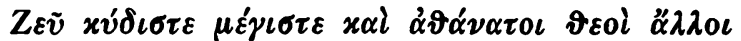

(Iliade $\Gamma 298$ ) - puis, dans la réponse de la sainte, le passage cor-

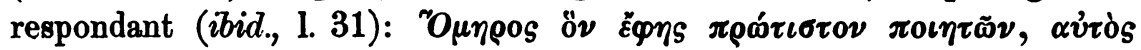

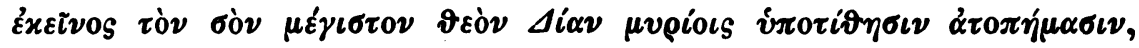

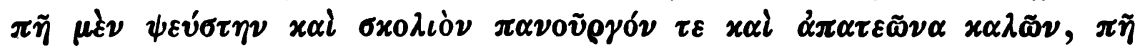

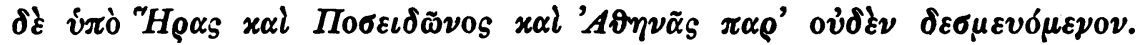

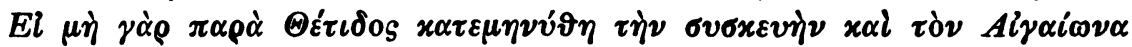

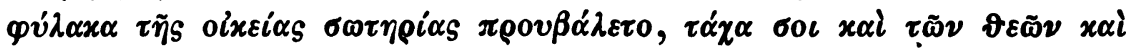

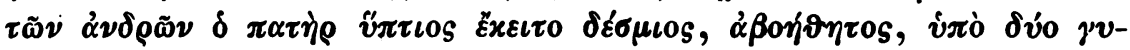

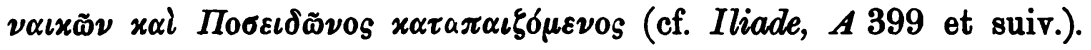

On a vu le problème se compliquer et la solution s'éloigner de nous à mesure que nous avancions: il $\mathrm{y}$ a des points de contact entre notre Passion et Malalas; il y en a aussi entre notre Passion et le recueil de Bentley; il y en a d'autres encore entre. le recueil de Bentley et Malalas; le recueil de Bentley n'est lui-même qu'un spécimen de toute une catégorie de recueils du même genre qui sont conservés dans nos manuscrits, et dont nous connaissons par hasard l'un ou

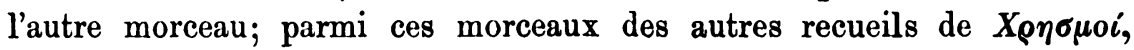
il en est qui coïncident à leur tour avec notre Passion; enfin, nous

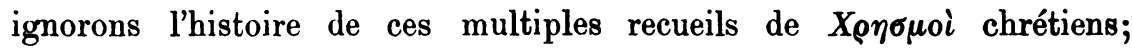
nous devinons à peine le nom (Aristokritos) de l'auteur de l'œuvre dont ces recueils pourraient être tous des extraits, des remaniements, ou des imitations, et le texte de Malalas lui-même ne nous est connu que d'une manière insuffisante. Il serait donc bien périlleux, pour le moment, de proposer un stemma, indiquant la parenté qui rattache

1) Il faut signaler - puisque Buresch ne l'a pas fait - que les $\$ 53-54$ de

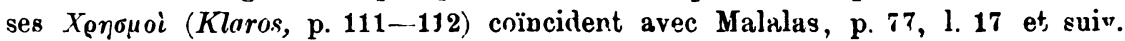
(Bonn). Y a-t-il d'autres rencontres du même genre entre les deux textes? il serait bon qu'on prît la peine de les chercher. 
notre Passion à ces divers documents. Faudrait-il supposer que Malalas a utilisé un recueil analogue à la @eoбoqia, peut-être même dérivé de l'œuvre d'Aristokritos; que le Malalas primitif en avait des extraits plus nombreux que l'édition de Bonn n'en contient, et que l'auteur de la Passion lui a emprunté toutes ou presque toutes ses citations? Cela parait fort douteux. N'est-ce pas plutôt de quelque collection de

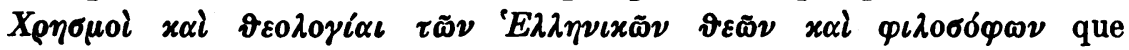
l'hagiographe s'est servi? Mais est-il possible de faire entrer dans un pareil recueil les dissertations variées qui ont été reproduites ci-dessus, et comment, avec cette hypothèse, faudrait-il expliquer l'étroite parenté des extraits de la Passion et des passages correspondants de Malalas? Devrait-on enfin supposer que l'auteur de la Passion a puisé ses citations à plus d'une source?

Bref, il y a là une question qu'il faut laisser à résoudre au futur

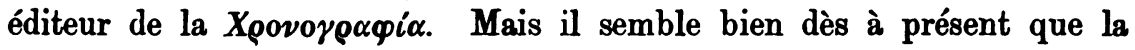
recherche des sources de Malalas ferait un grand progrès, si l'on se

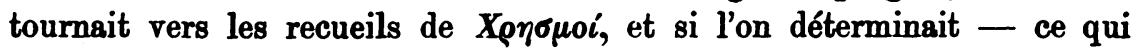
pourrait se faire avec assez de précision - de quelle collection Malalas s'est servi; pour Aristokritos aussi, il importerait que l'on cherchât à savoir au plus tôt si des éditions incomplètes et fortement altérées de sa - Oroбoqía circulaient dès les temps de Malalas. Enfin, en ce qui concerne l'hagiographe lui-même, on arrive à un résultat certain: il paraît avoir lu philosophes et poètes, mais il n'y a là qu'une science d'emprunt, et ses citations proviennent d'un plagiat.

J. Bidez. 